\title{
YRNA expression predicts survival in bladder cancer patients
}

\author{
Yuri Tolkach ${ }^{1 \dagger}$, Anna Franziska Stahl ${ }^{2 \dagger}$, Eva-Maria Niehoff ${ }^{2}$, Chenming Zhao ${ }^{2}$, Glen Kristiansen ${ }^{1}$, \\ Stefan Cajetan Müller ${ }^{2}$ and Jörg Ellinger ${ }^{2^{*}}$ (i)
}

\begin{abstract}
Background: Non-coding RNAs play an important role in human carcinogenesis. YRNAs (Ro-associated Y), a novel class of non-coding RNAs, have been identified as biomarker in various malignancies, but remain to be studied in urinary bladder cancer (BCA) patients.

Methods: The expression of all four YRNAs (RNY1, RNY3, RNY4, RNY5) was determined in archival BCA (urothelial carcinoma, $n=88$ ) and normal urothelial bladder $(n=30)$ tissues using quantitative real-time PCR. Associations with clinicopathological parameters and prognostic role for overall and cancer-specific survival were analysed.

Results: All YRNAs were significantly downregulated in BCA tissue. A low expression of RNY1, RNY3 and RNY4 was associated with muscle-invasive BCA, lymph node metastases and advanced grade. Furthermore, expression of RNY1 and RNY3 was predictive for BCA patients' overall (also RNY4) and cancer-specific survival as estimated using Kaplan-Meier and univariate (but not multivariate) Cox regression analyses. RNY1, RNY3 and RNY4 show good discriminative ability between tumor and normal tissue, as well as between muscle-invasive and non-muscleinvasive urothelial carcinoma.
\end{abstract}

Conclusions: The expression of YRNAs is altered in BCA and associated with poor prognosis. Possible diagnostic role of YRNAs should be investigated in further studies.

Keywords: YRNA, Biomarker, Bladder cancer, Prognosis

\section{Background}

Urinary bladder cancer (BCA) is among the most common malignancies worldwide; approximately 430.000 new cases and 165.000 deaths were estimated for 2012 [1]. An important step in BCA progression is the invasion of the detrusor muscle and metastatic spread. BCA symptoms are sometimes non-specific leading to delayed diagnoses at an invasive stage, which is accompanied with an unfavorable outcome. To improve the therapeutic management a better understanding of the molecular biology of BCA is necessary.

The vast majority of the human genome (98\%) consists of non-coding genes [2]. Non-coding RNA (ncRNAs) do not encode proteins, but have a putative regulative func-

\footnotetext{
* Correspondence: joerg.ellinger@ukbonn.de

${ }^{\dagger}$ Equal contributors

${ }^{2}$ Department of Urology, University Hospital Bonn, Bonn, Germany

Full list of author information is available at the end of the article
}

tion of gene expression. The ncRNAs are classified according to their size in nucleotides (nt) into smallncRNAs (sncRNA <200 nt) and long-ncRNAs (lncRNA $>200 \mathrm{nt}$ ) [3]. Much effort has been spent to identify and functionally characterize dysregulated microRNAs $[4,5]$ and lncRNAs [6] in BCA in the past years, but few is known about other subtypes of the ncRNAs. YRNAs (Ro-associated Y) were recognized as a component of soluble ribonucleoproteins (Ro RNPS) in the blood of patients with rheumatic autoimmune diseases [7]. Nowadays, four highly conserved human YRNAs (RNY1, RNY3, RNY4, and RNY5) are known. YRNAs have a size of $80-110 \mathrm{nt}$ and a stem-loop structure due to their complementary $5^{\prime}$ and $3^{\prime}$ ends [8]. They are functionally relevant for DNA replication [9] and Ro60 inhibition [10]. YRNAs are overexpressed in various cancer cells [11], and RNY1 and RNY3 inhibition was shown to decrease cell proliferation [11, 12]. YRNA-derived fragments are involved in caspase-3-dependent cell death 
and NF-kB-dependent inflammation and may have an inflammatory role [13]. It was also shown that RNY5 fragments in extracellular vesicles trigger cell death, and thereby may help cancer cells to optimize the microenvironment for proliferation and invasion [14]. YRNAs have not been investigated in a large cohort of BCA so far; we therefore studied the expression profile of YRNAs in BCA and normal urothelial tissue.

\section{Methods \\ Patients}

Formalin-fixed, paraffin embedded (FFPE) bladder tissues were randomly selected from the archive of the Institute of pathology at the University Hospital Bonn from patients $(n=112)$ who underwent transurethral resection of the bladder (TURB) or radical cystectomy for BCA from 1990 until 2009. Follow-up information was available for all patients; median follow-up time was 51 months (range 1-210). The detailed clinicopathological parameters are reported in Table 1.

\section{Ethics, consent and permissions}

All patients gave written informed consent for the collection of biomaterials within the framework of the Biobank at the University Hospital Bonn. The study was

Table 1 Clinicopathological characteristics of the study cohort

\begin{tabular}{lll}
\hline & Normal & BCA \\
& $n=30$ & $n=88$ \\
\hline Sex & $22(73 \%)$ & $70(79.5 \%)$ \\
Male & $8(27 \%)$ & $18(20.5 \%)$ \\
Female & & \\
Age & 66.3 & 69.1 \\
Mean & $43-81$ & $40-91$ \\
Range & & \\
Staging & & $13(14.8 \%)$ \\
pT-stage & n.a. & $13(14.8 \%)$ \\
pTa & n.a. & $18(20.5 \%)$ \\
pTis & n.a. & $12(13.6 \%)$ \\
pT1 & n.a. & $15(17.0 \%)$ \\
pT2 & n.a. & $17(19.3 \%)$ \\
pT3 & n.a. & $23(26.1 \%)$ \\
pT4 & n.a. & $2(2.3 \%)$ \\
Lymph node metastasis & n.a. & $9(10.2 \%)$ \\
Distant metastasis & & $31(35.2 \%)$ \\
Grading & n.a. & \\
grade 1 & n.a. & \\
grade 2 & n.a. & \\
\hline
\end{tabular}

approved by the ethic committee $(280 / 12)$ at the University Hospital Bonn.

\section{Tissue samples acquisition}

A first $5 \mu \mathrm{m}$ thick section from the FFPE block was stained with haematoxylin and eosin and used for histological control and mapping of the block content. BCA $(n=88)$ and normal urothelial tissue $(n=30)$ samples were then macrodissected using a scalpel from five consecutive $20 \mu \mathrm{m}$ sections of the block. The absence of significant inflammation as well as absence of any signs of dysplasia/atypia was ensured morphologically in normal tissues. Some samples with normal urothelial tissue stemmed from patients with BCA, when spatial divergence of these samples could be guaranteed and carcinoma in situ did not coexist.

\section{RNA isolation and quantitative real-time PCR}

The RNA was isolated using the Recover All Total Nucleic Acid Isolation Kit (Ambion, Foster City, CA, USA) according to the suppliers recommendations. Afterwards, the DNA-free DNA Removal Kit (Ambion) was used to digest DNA contaminants. RNA purity and concentration were determined using the NanoDrop 2000 spectrophotometer (Thermo Scientific, Wilmington, DE, USA). The isolated total RNA was stored at $-80{ }^{\circ} \mathrm{C}$ until further use.

cDNA synthesis and real-time PCR were performed as described in detail by Nientiedt et al. [12], adopted for FFPE tissues. In brief, reverse transcription was carried out with the miScript II RT Kit (Qiagen, Hilden, Germany). Then, $1 \mathrm{ng}$ cDNA template was used for quantification using the Qiagen miScript SYBR Green PCR technology (Hilden, Germany). Self-designed primers were used for the quantification of YRNAs (RNY1 forward: GGC-TGG-TCC-GAA-GGT-AGT-GAG; RNY1 reverse: GGG-GGA-AAG-AGT-AGA-ACA-AGG; RNY3 forward: CCG-AGT-GCA-GTG-GTG-TTT-AC; RNY3 reverse: AAG-CAG-TGG-GAG-TGG-AGA-A; RNY4 forward: TCC-GAT-GGT-AGT-GGG-TTA-TCA; RNY4 reverse: AAA-GCC-AGT-CAA-ATT-TAG-CAG-T. The primer design was performed using Primer-BLAST [15]. The RNY5 primer was published by Christov et al. [9].. YRNA expression levels were normalized to SNORD43 (Qiagen miScript Primer Assay: MS00007476) and RNU6-2 (Qiagen miScript Primer Assay: MS00007476), earlier shown to be a suitable reference gene for urological malignancies [5, 16]. PCR experiments were carried out on a Quant Studio 5 Real-Time PCR System (Applied Biosystems, Foster City, CA, USA). Relative YRNA expression levels were calculated using the $2^{-\Delta \Delta \mathrm{CT}}$ algorithm; the PCR efficiencies were: RNY1 101.0\%, RNY3 95.3\%, RNY4 95.7\%, RNY5 104.7\%. Each PCR assay included multiple 
control samples (no-RT-sample, genomic DNA, no template control, RT4 cell line RNA as positive control).

\section{Statistics}

Statistical analyses were performed $\mathrm{R}$ ( $\mathrm{R}$ Foundation for Statistical Computing; version 3.3.3). The Mann-WhitneyWilcoxon test was used to compare YRNA expression in subgroups. The Spearman-Rho test was used to correlate clinical parameters and YRNA expression. Kaplan-Meier curves, log-rank test, univariate and multivariate Cox proportional hazards regression analysis were used for survival analyses. pROC-package for $\mathrm{R}$ was used for ROCAnalyses. survMisc-package was used for selection of the best cutoff during survival analyses.

\section{Results}

Firstly, we have compared the YRNA expression levels in normal and malignant bladder tissues. Median expression levels (Table 2) were significantly (all $p<0.001$ ) lower in BCA tissue than in normal tissue (RNY1: 0.59 in normal vs 0.15 in BCA; RNY3: 0.84 in normal vs 0.21 in BCA; RNY4: 2.92 in normal vs 0.61 in BCA; and RNY5: 2.34 in normal vs 1.15 in BCA). As determined using ROC analyses (Fig. 1a, Table 2), the tissue analysis of YRNAs allowed discrimination of BCA tumor tissue and normal urothelial mucosa with an area under curve of 0.715 (RNY5) up to 0.863 (RNY3). YRNA expression levels were correlated with each other (all $p<0.001$, Additional file 1: Figure S1): especially RNY1, RNY3 and RNY4 expression was highly correlated $\left(r^{2}>0.87\right)$, whereas RNY5 levels were less distinctly correlated to RNY1 $\left(r^{2}=0.45\right)$, RNY3 $\left(r^{2}=0.40\right)$ and RNY4 $\left(r^{2}=0.66\right)$.

Furthermore, we analyzed, whether differences in YRNA expression were associated with adverse clinicopathological parameters (Additional files 2, 3 and 4: Tables S1-S3). RNY1-, RNY3- and RNY4-expression (all $p<0.001)$ was significantly decreased in muscle-invasive BCA (MIBC) compared to non-muscle-invasive BCA (NMIBC), whereas RNY5 expression levels in those were similar $(p=0.739)$ (Fig. 1c-f). Discrimination of the MIBC and NMIBC tumors was possible with maximal AUC of 0.780 for RNY3 (Fig. 1b). The expression of
RNY1 $(p=0.011)$, RNY3 $(p<0.001)$ and RNY4 $(p=0.041)$ was also lower in high grade (G3) compared to lower grade (G1 and G2) tumors; RNY5 ( $p=0.877$ ) expression levels were not correlated with grading. Presence of lymph node metastases was also associated with decreased RNY1 $(p<0.001)$, RNY3 $(p<0.001)$ and RNY4 ( $p=0.007)$ expression. YRNA expression was not correlated with age nor gender (all $p>0.2$ ).

Finally, the relevance of YRNAs for patients' prognosis was determined using Kaplan Meier estimates. Expression of YRNA was significantly correlated with BCA patients' overall (RNY1, RNY3, RNY4) and cancerspecific (RNY1, RNY3) survival (all log rank $p<0.05$; Fig. 2). We also performed univariate and multivariate Cox regression analyses: RNY1 and RNY3 were significantly predictive for cancer-specific and overall survival (all $p<0.05$ ), but lost their predictive value in a multivariate model (see Tables 3 and 4 for details).

\section{Discussion}

YRNAs have been identified as novel non-coding class of RNA molecules which may be used as biomarker for cancer [17-19]. So far, little information exists about the expression of YRNA in BCA patients. In 2008, Christov et al. demonstrated an increase of YRNA expression [11]. However, his study cohort was very small $(n=4)$ and thereby limiting any meaningful statistical conclusion. Thus, we investigated the expression of all four YRNAs in an enlarged cohort of BCA patients to allow a robust statistical analysis. Interestingly, YRNA expression levels were significantly downregulated in our dataset, mean expression levels in BCA tissue were 2- to 4-fold lower than in normal tissue. It should be noted that Christov et al. [11] normalized the YRNA expression to the mRNA HPRT1, whereas our study used RNU6-2 and SNORD43; RNU6-2 and SNORD43 were earlier shown to be useful reference genes for the analysis of BCA samples [16]. Notably, as expected from the experiments of Christov et al. [11], RNY1, RNY3 and RNY4 expression was highly correlated, whereas the degree of correlation of RNY5 to the other YRNAs was less pronounced.

Table 2 Relative YRNA expression levels in the tumor and normal urothelial tissue and discriminative capabilities of the YRNAs to predict the tissue dignity (tumor vs. normal)

\begin{tabular}{|c|c|c|c|c|c|c|c|}
\hline & \multicolumn{2}{|c|}{ Expression median (range) } & \multicolumn{2}{|c|}{ ROC analysis } & \multirow[t]{2}{*}{ Sensitivity } & \multirow[t]{2}{*}{ Specificity } & \multirow[t]{2}{*}{ Cut-off } \\
\hline & $\mathrm{BCA}(n=88)$ & CTRL $(n=30)$ & AUC & $95 \% \mathrm{Cl}$ & & & \\
\hline RNY1 & $0.15(0.0-1.75)$ & $0.59(0.02-2.54)$ & 0.851 & $0.760-0.941$ & $73.3 \%$ & $90.9 \%$ & 0.471 \\
\hline RNY3 & $0.21(0.0-1.96)$ & $0.84(0.03-3.24)$ & 0.863 & $0.778-0.949$ & $80.0 \%$ & $88.6 \%$ & 0.527 \\
\hline RNY4 & $0.61(0.0-8.96)$ & $2.92(0.05-18.41)$ & 0.844 & $0.755-0.933$ & $86.7 \%$ & $75.0 \%$ & 1.061 \\
\hline RNY5 & $1.15(0.0-8.41)$ & $2.34(0.07-18.75)$ & 0.715 & $0.601-0.829$ & $73.3 \%$ & $75.0 \%$ & 1.948 \\
\hline
\end{tabular}

Comments: BCA - tissue samples with urothelial cancer (bladder cancer); CTRL - control samples (normal urothelial tissue); AUC, area under the curve; $95 \%$ Cl, $95 \%$ confidence interval 


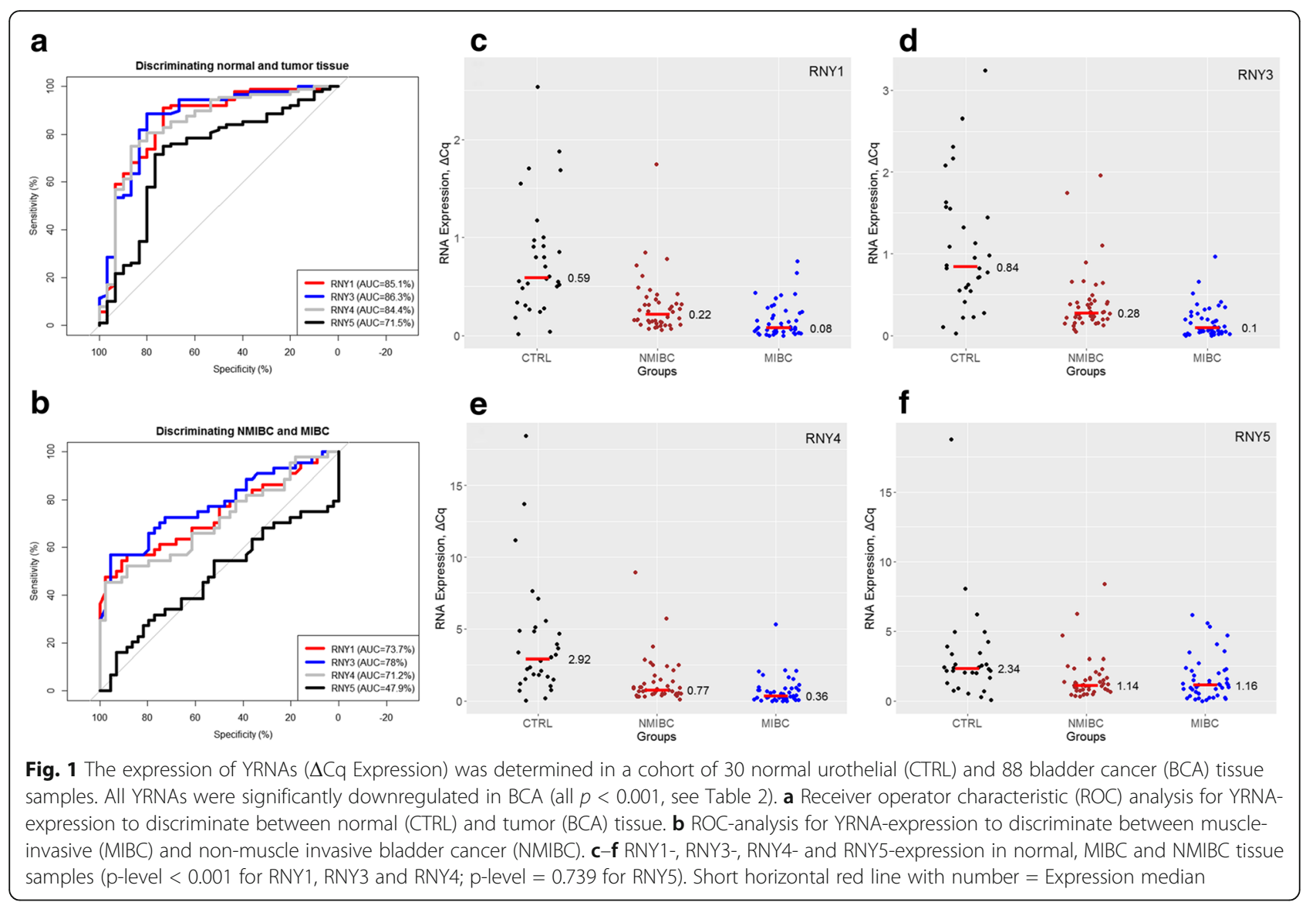

The analysis of 88 tissue samples with urothelial carcinoma allowed as to correlate the expression of YRNAs with clinicopathological parameters. RNY1, RNY3 and RNY4 expression was associated with advanced stage (muscle invasive BCA, lymph node metastasis) and grade (G3 tumors when opposed to G1 and G2). Importantly, the expression levels of RNY1 and RNY3 were significantly predictive for cancer-specific and overall survival of BCA patients with a clear trend for RNY4. However, the strong correlation of YRNA expression and muscle-invasiveness of the tumor impaired achieving an independency in the multivariate Cox regression analyses within a cohort of 88 BCA patients.

Although YRNA are non-coding RNAs, they are also of functional relevance and do not represent transcriptory garbage. YRNAs are essential factors for chromosomal DNA replication [9], whereby they execute their function during the initiation of DNA replication [20]. siRNA mediated knock-down of RNY1 and RNY3 reduced the number proportion of $\mathrm{S}$ phase cells in the HeLa cells; degradation of RNY3 reduced also the number S-phase cells in EJ30 bladder cancer cells. Furthermore, the mitotic index and the cell density was reduced after treatment with RNY3 siRNAs [11]. Within this context it is interesting that we have observed a decrease of YRNA levels in BCA patients. Seemingly, Similar trends with decreased abundance of several YRNAs in tumor patients (in serum) were observed in other tumor types (head and neck squamous cell carcinomas [18], breast cancer [19]), which support our findings, even given the fact that conclusions from cell line studies are suggesting the upregulation could be associated with tumor growth and proliferation This could be related, from one side, to different YRNA effects in different primary tumors and, from the other side, to the artificial construct and well known limitations of cell cultures. Also, the effect of YRNA overexpression was not studied in the above mentioned cell culture study [11]. The biological functions of the YRNA are still understudied and could be multidirectional. Some studies show that YRNAs demonstrating decreased levels during mitosis and high levels during $S$ and G2 phases of the cell cycle, partially through association with chromatin [21]. This may be a possible explanation for decreased expression in highly proliferating tumor tissues. Many microRNAs are known for "managing" the cell fate and cell proliferation through interactions with p53 and other members of p53-family [22], which is highly deregulated in tumor 

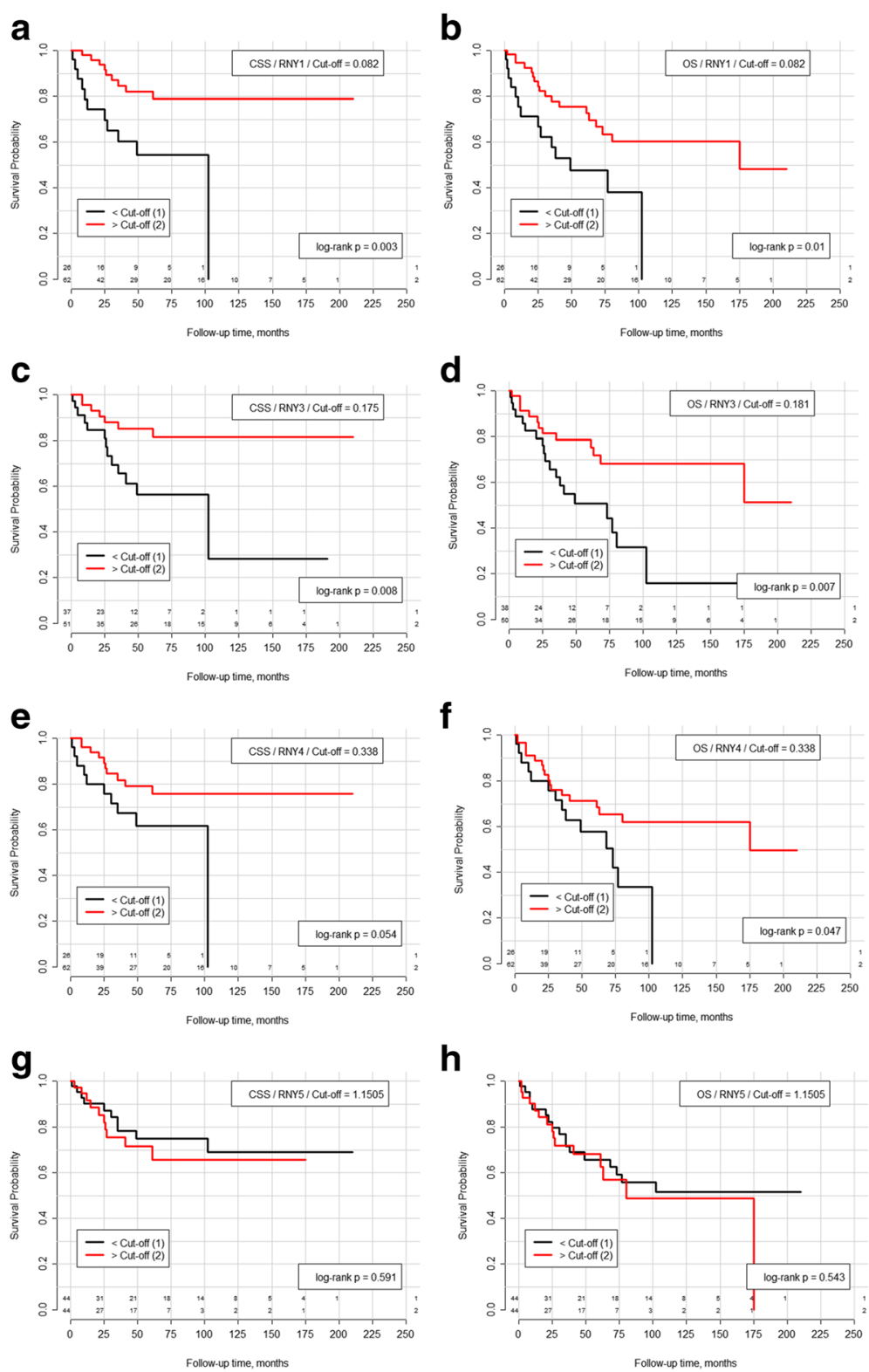

Fig. 2 Kaplan-Meier curves and log-rank test for YRNAs expression dichotomized based on the best cut-off, in each case separately for cancerspecific and overall survival, respectively: a/b RNY1, c/d RNY3, e/f RNY4, and $\mathbf{g} / \mathbf{h}$ RNY5. Kaplan Meier estimates indicate that expression of YRNAs is statistically significant prognostic for cancer-specific survival (RNY1, RNY3) and overall survival (RNY1, RNY3, RNY4) in BCA patients (all log-rank $p>0.05)$. Abbreviations: OS - overall survival, CSS - cancer-specific survival

tissue of patients with urinary bladder cancer compared to normal tissue [23]. The interactions with this pathway were not studied for YRNA to date and would probably also provide the explanations for aberrant YRNA expression. It may also be speculated that YRNAs are secreted by BCA cells to act as mediator of immunoescape: extracellular YRNAs fragments activate TLR7 to promote apoptosis in macrophages and monocytes [13].

YRNAs are expected to be a suitable non-invasive biomarker because approximately 25 to 33 nt large YRNA fragments have been identified using small RNA sequencing in human serum and plasma [17]. It was further shown that changes of specific YRNA fragments in serum are associated with ER-negative breast cancer [19]. Similarly, specific YRNA fragments were also circulating at altered levels in head and neck cancer patients [18]. However, specific identification of these small ( 25 to $33 \mathrm{nt}$ size) fragments implies application of small RNA sequencing procedures and is therefore at least today not suited for daily routine. In our study we were able for the first time to show that RNY1, 
Table 3 Cox regression analysis for the prediction of cancer-specific survival in patients with urothelial bladder cancer $(n=88$, number of events $=20$ )

\begin{tabular}{|c|c|c|c|c|c|c|}
\hline & Univariat & & & Multivari & & \\
\hline & $p$-value & $\mathrm{HR}$ & $95 \% \mathrm{Cl}$ & $p$-value & $H R$ & $95 \% \mathrm{Cl}$ \\
\hline RNY1, low vs high & 0.005 & 3.57 & $1.46-8.72$ & 0.676 & 1.22 & $0.47-3.17$ \\
\hline RNY3, low vs high & 0.012 & 3.29 & $1.29-8.35$ & 0.715 & 1.20 & $0.45-3.25$ \\
\hline RNY4, low vs high & 0.062 & 2.34 & $0.96-5.69$ & 0.816 & 0.90 & $0.35-2.27$ \\
\hline RNY5, low vs high & 0.593 & 0.79 & $0.32-1.90$ & 0.494 & 0.73 & $0.30-1.80$ \\
\hline MIBC vs. NMIBC & $6.7 e-04$ & 12.89 & $2.95-56.26$ & 0.017 & $7.97^{*}$ & $1.45-43.71$ \\
\hline pN-stage, pN1 vs pN0 & 0.001 & 4.38 & $1.81-10.59$ & 0.741 & $1.19^{*}$ & $0.43-3.29$ \\
\hline cM-stage, M1 $(n=2)$ vs M0 & $2.9 e-04$ & 21.2 & $4.06-110.8$ & $x$ & $x$ & $x$ \\
\hline Grade, G3 vs G1 + G2 & 0.006 & 4.72 & $1.57-14.21$ & 0.347 & $1.82^{*}$ & $0.52-6.35$ \\
\hline
\end{tabular}

Comments: ${ }^{\text {In }}$ case of every YRNA data is shown for separate multivariate model with "MIBC vs NMIBC", $\mathrm{pN}$-Stage and Grade as co-variates; *HR, $p$-value, $95 \%$ CI shown for multivariate model with RNY1. Other YRNAs (RNY3, RNY4, RNY5) showed similar results

Analysis for YRNAs is based on the best cut-off, the same as in Kaplan-Meier/log-rank analysis: RNY1-0.082, RNY3-0.175, RNY4-0.338, RNY5-1.151. Abbreviations: $M I B C$ muscle-invasive bladder cancer, NMIBC non-muscle-invasive bladder cancer

RNY3 and RNY4 could very good discriminate between the normal and tumor tissue with a maximal AUC of 0.863 (RNY3), and to a lesser extent between muscle-invasive and not-muscle-invasive tumors (maximal AUC 0.780 for RNY3). These findings could support the diagnostic value of YRNA, which certainly warrants further investigations.

Some limitations of our study should be acknowledged: The RNA integrity was not determined after RNA isolation. Formalin-fixed, paraffin embedded tissues are usually degraded to approximately $>200-400 \mathrm{bp}$ sized RNA fragments [24, 25], and thus amplifying PCR products of approximately $100 \mathrm{bp}$ size is feasible. We randomly picked samples obtained over a period of approximately 20 years and long-term storage may alter the RNA integrity [26]. However, relative YRNA expression levels were not correlated with the year of surgery (data not shown). The normal urothelial tissue samples were in several patients derived from patients with BCA, however necessary precautions were undertaken to prevent contamination of normal samples with tumor tissue (see Materials and methods). Although even in this case we cannot exclude molecular alterations occurred in the microscopically normal urothelium. The tissue was macrodissected with a scalpel, thus RNA some degree of inevitable contamination with other cells like inflammatory, stromal or endothelial cells could have affected the YRNA expression studies.

\section{Conclusions}

The expression of all four YRNAs is downregulated in tumor tissue in patients with urinary bladder urothelial carcinoma. Expression changes are associated with advanced disease, higher grade and metastatic disease and may have prognostic relevance for cancer-specific and overall survival.

Table 4 Cox regression analysis for the prediction of overall survival in patients with urothelial bladder cancer $(n=88$, number of events $=32$ )

\begin{tabular}{|c|c|c|c|c|c|c|}
\hline \multirow[t]{2}{*}{ Overall Survival } & \multicolumn{3}{|c|}{ Univariate analysis } & \multicolumn{3}{|c|}{ Multivariate analysis $^{a}$} \\
\hline & $p$-value & $H R$ & $95 \% \mathrm{Cl}$ & $p$-value & $\mathrm{HR}$ & $95 \% \mathrm{Cl}$ \\
\hline RNY1, low vs high & 0.013 & 2.49 & $1.22-5.08$ & 0.615 & 1.24 & $0.55-2.80$ \\
\hline RNY3, low vs high & 0.009 & 2.60 & $1.27-5.33$ & 0.400 & 1.43 & $0.62-3.26$ \\
\hline RNY4, low vs high & 0.053 & 2.03 & $0.99-4.15$ & 0.847 & 1.08 & $0.49-2.41$ \\
\hline RNY5, low vs high & 0.545 & 0.80 & $0.40-1.63$ & 0.531 & 0.80 & $0.39-1.62$ \\
\hline MIBC vs. NMIBC & $4.9 e-04$ & 4.05 & $1.85-8.90$ & 0.055 & $2.86^{*}$ & $0.98-8.36$ \\
\hline pN-stage, pN1 vs pN0 & 0.003 & 2.89 & $1.44-5.84$ & 0.641 & $1.24^{*}$ & $0.50-3.05$ \\
\hline cM-stage, M1 (n=2) vs M0 & 0.001 & 12.87 & $2.71-61.16$ & $x$ & $x$ & $x$ \\
\hline Grade, G3 vs G1 + G2 & 0.021 & 2.39 & $1.14-5.01$ & 0.575 & $1.29 *$ & $0.55-3.09$ \\
\hline
\end{tabular}

Comments: ${ }^{2}$ In case of every YRNA data is shown for separate multivariate model with "MIBC vs NMIBC", $\mathrm{pN}-\mathrm{Stage}$ and Grade as co-variates; ${ }^{*} \mathrm{HR}, p$-value, $95 \%$ Cl shown for multivariate model with RNY1. Other YRNAs (RNY3, RNY4, RNY5) showed similar results

Analysis for YRNAs is based on the best cut-off, the same as in Kaplan-Meier/log-rank analysis: RNY1-0.082, RNY3-0.181, RNY4-0.338, RNY5-1.151. Abbreviations: $M I B C$ muscle-invasive bladder cancer, NMIBC non-muscle-invasive bladder cancer 


\section{Additional files}

Additional file 1: Figure S1. Correlation matrix (Spearman rho) between the expression of different YRNAs (PCR, $\triangle$ Cq Expression) in tumor tissue (all $p<0.001$ ). (DOCX $29 \mathrm{~kb}$ )

Additional file 2: Table S1. Expression of RNYs (PCR, $\Delta C q$ expression): non-muscle-invasive bladder cancer (NMIBC) vs muscle-invasive bladder cancer (NMIBC). (DOCX 15 kb)

Additional file 3: Table S2. Expression of RNYS (PCR, $\triangle$ Cq expression): patients with lymph node metastases $(\mathrm{pN}+)$ vs patients without lymph node metastases (pNO). (DOCX $15 \mathrm{~kb}$ )

Additional file 4: Table S3. Expression of RNYS (PCR, $\triangle$ Cq expression): patients stratified according to grade of tumor differentiation. (DOCX $15 \mathrm{~kb}$ )

\section{Abbreviations}

BCA: Bladder cancer; FFPE: Formalin-fixed, paraffin embedded; ncRNA: noncoding RNA; YRNA: Ro-associated Y RNA

\section{Acknowledgments}

We thank Doris Schmidt for technical assistance.

\section{Funding}

No funding was received.

\section{Availability of data and materials}

The datasets used and/or analyzed during the current study are available from the corresponding author on reasonable request.

\section{Authors contributions}

Study concepts and design: JE, SCM; Data acquisition: AFS, EMN, CZ; Sample classification and acquisition: JE, GK, SCM; Analysis and interpretation of data: $Y T$, AFS, EMN, JE; Drafting of manuscript: YT, JE; Supervising manuscript critically for important intellectual content: EMN, CZ, GK, SCM. Approval of final manuscripts: all authors.

\section{Ethics approval and consent to participate}

All patients gave written informed consent for the collection of biomaterials within the framework of the Biobank at the University Hospital Bonn. The study was approved by the Ethikkommission at the Medical Faculty at the University Bonn (approval number 280/12)

\section{Consent for publication}

Not applicable

\section{Competing interests}

The authors declare that they have no competing interests.

\section{Publisher's Note}

Springer Nature remains neutral with regard to jurisdictional claims in published maps and institutional affiliations.

\section{Author details}

${ }^{1}$ Institute of Pathology, University Hospital Bonn, Bonn, Germany.

${ }^{2}$ Department of Urology, University Hospital Bonn, Bonn, Germany.

Received: 21 May 2017 Accepted: 31 October 2017

Published online: 10 November 2017

\section{References}

1. Antoni S, Ferlay J, Soerjomataram I, Znaor A, Jemal A, Bray F. Bladder cancer incidence and mortality: a global overview and recent trends. Eur Urol. 2017:71(1):96-108

2. Wilusz JE, Sunwoo H, Spector DL. Long noncoding RNAs: functional surprises from the RNA world. Genes Dev. 2009;23(13):1494-504.

3. Martens-Uzunova ES, Olvedy M, Jenster G. Beyond microRNA-novel RNAs derived from small non-coding RNA and their implication in cancer. Cancer Lett. 2013;340(2):201-11.
4. Ellinger J, Muller SC. MicroRNAs: a novel non-invasive biomarker for patients with urological malignancies. Curr Pharm Biotechnol. 2014;15(5):486-91.

5. Eissa S, Matboli M, Hegazy MG, Kotb YM, Essawy NO. Evaluation of urinary microRNA panel in bladder cancer diagnosis: relation to bilharziasis. Transl Res. 2015;165(6):731-9.

6. Chen J, Miao Z, Xue B, Shan Y, Weng G, Shen B. Long non-coding RNAs in urologic malignancies: functional roles and clinical translation. J Cancer. 2016;7(13):1842-55.

7. Hendrick JP, Wolin SL, Rinke J, Lerner MR, Steitz JA. Ro small cytoplasmic ribonucleoproteins are a subclass of la ribonucleoproteins: further characterization of the Ro and la small ribonucleoproteins from uninfected mammalian cells. Mol Cell Biol. 1981;1(12):1138-49.

8. Pruijn GJ, Wingens PA, Peters SL, Thijssen JP, van Venrooij WJ. Ro RNP associated $Y$ RNAs are highly conserved among mammals. Biochim Biophys Acta. 1993:1216(3):395-401.

9. Christov CP, Gardiner TJ, Szuts D, Krude T. Functional requirement of noncoding $Y$ RNAs for human chromosomal DNA replication. Mol Cell Biol. 2006;26(18):6993-7004

10. Stein AJ, Fuchs G, Fu C, Wolin SL, Reinisch KM. Structural insights into RNA quality control: the Ro autoantigen binds misfolded RNAs via its central cavity. Cell. 2005;121(4):529-39.

11. Christov CP, Trivier E, Krude T. Noncoding human Y RNAs are overexpressed in tumours and required for cell proliferation. Br J Cancer. 2008;98(5):981-8.

12. Nientiedt M, Schmidt D, Kristiansen G, Muller SC, Ellinger J. YRNA expression profiles are altered in clear cell renal cell carcinoma. Eur Urol Focus. 2016; (16):30112-2.

13. Hizir Z, Bottini S, Grandjean V, Trabucchi M, Repetto E. RNY (YRNA)-derived small RNAs regulate cell death and inflammation in monocytes/ macrophages. Cell Death Dis. 2017;8(1):e2530.

14. Chakrabortty SK, Prakash A, Nechooshtan G, Hearn S, Gingeras TR. Extracellular vesicle-mediated transfer of processed and functional RNY5 RNA. RNA (New York, NY). 2015;21(11):1966-79.

15. Ye J, Coulouris G, Zaretskaya I, Cutcutache I, Rozen S, Madden TL. PrimerBLAST: a tool to design target-specific primers for polymerase chain reaction. BMC bioinformatics. 2012:13:134

16. Sanders I, Holdenrieder S, Walgenbach-Brunagel G, von Ruecker A, Kristiansen G, Muller SC, Ellinger J. Evaluation of reference genes for the analysis of serum miRNA in patients with prostate cancer, bladder cancer and renal cell carcinoma. Int J Urol. 2012;19(11):1017-25.

17. Dhahbi JM, Spindler SR, Atamna H, Boffelli D, Mote P, Martin DI. 5'-YRNA fragments derived by processing of transcripts from specific YRNA genes and pseudogenes are abundant in human serum and plasma. Physiol Genomics. 2013;45(21):990-8.

18. Victoria Martinez B, Dhahbi JM, Nunez Lopez YO, Lamperska K, Golusinski P, Luczewski L, Kolenda T, Atamna H, Spindler SR, Golusinski W, et al. Circulating small non-coding RNA signature in head and neck squamous cell carcinoma. Oncotarget. 2015;6(22):19246-63.

19. Dhahbi JM, Spindler SR, Atamna H, Boffelli D, Martin DI. Deep sequencing of serum small RNAs identifies patterns of $5^{\prime}$ tRNA half and YRNA fragment expression associated with breast cancer. Biomarkers in cancer. 2014;6:37-47.

20. Krude T, Christov CP, Hyrien O, Marheineke K. Y RNA functions at the initiation step of mammalian chromosomal DNA replication. J Cell Sci. 2009; 122(Pt 16):2836-45

21. Kheir E, Krude T. Non-coding Y RNAs associate with early replicating euchromatin in concordance with the origin recognition complex. J Cell Sci. 2017;130(7):1239-50

22. Liu J, Zhang C, Zhao Y, Feng Z. MicroRNA control of p53. J Cell Biochem. 2017;118(1):7-14.

23. Ciccarese C, Massari F, Blanca A, Tortora G, Montironi R, Cheng L, Scarpelli M, Raspollini MR, Vau N, Fonseca J, et al. Tp53 and its potential therapeutic role as a target in bladder cancer. Expert Opin Ther Targets. 2017;21(4):401-14.

24. Ludyga N, Grunwald B, Azimzadeh O, Englert S, Hofler H, Tapio S, Aubele M. Nucleic acids from long-term preserved FFPE tissues are suitable for downstream analyses. Virchows Archiv : an international journal of pathology. 2012;460(2):131-40

25. von Ahlfen S, Missel A, Bendrat K, Schlumpberger M. Determinants of RNA quality from FFPE samples. PLoS One. 2007;2(12):e1261

26. Hall JS, Taylor J, Valentine HR, Irlam JJ, Eustace A, Hoskin PJ, Miller CJ, West CM. Enhanced stability of microRNA expression facilitates classification of FFPE tumour samples exhibiting near total mRNA degradation. Br J Cancer. 2012;107(4):684-94. 